\title{
Gentechnisch verånderte Pflanzen der "Zweiten und Dritten Generation": Was können wir erwarten?
}

\section{Zusammenfassung}

Die gentechnisch veränderten Pflanzen werden aufgrund ihrer gentechnischen Veränderungen klassifiziert und fünf verschiedenen Anwendungsgebieten zugeordnet. Während die gentechnisch veränderten Pflanzen des Anwendungsgebietes „Agronomischer Bedarf" (z.B. Herbizid-Toleranz und InsektenResistenz) bereits in zahlreichen Freilandversuchen im Bereich der EU-Mitgliedstaaten getestet wurden und für einige dieser gentechnisch veränderten Pflanzen (und der aus innen hergestellten Produkte) auch schon EU-weit gültige Genehmigungen für ihr Inverkehrbringen vorliegen, folgen die gentechnisch veränderten Pflanzen der „Zweiten und Dritten Generation" zeitlich um einige Jahre verzögert dieser Entwicklung nach. Es handelt sich bei ihnen um die Anwendungsbereiche „Endogene Inhaltstoffe", "Neuartige Inhaltstoffe für die Lebensmittelproduktion, ,,Pharmazeutisch/medizinische Anwendungen” sowie „Anwendungen für die chemische Industrie".

Aber nicht nur die Palette der möglichen gentechnischen Veränderungen wird sich zukünftig bei den gentechnisch veränderten Pflanzen erweitern sowie sich schwerpunktmäßig mehr vom ersten Anwendungsbereich auf die anderen vier verlagern, sondern auch das grundsätzliche gentechnische Design der gentechnisch veränderten Pflanzen wird sich durch den Einsatz der Chloroplastentransformation, der Chimeraplastie sowie der "Genetic Use Restriction Technology" (GURT) fortentwickeln.

\section{Schlüsselwörter}

Endogene Inhaltsstoffe • Neuartige Inhaltsstoffe · Pharmazeutisch/medizinische Anwendungen · Chimeraplastie · GURT

n den letzten Jahren ist der Markt für Produkte, die mittels gentechnologischer Methoden erzeugt wurden, beachtlich gewachsen; 1998 betrug der Marktwert der weltweit in den Handel gebrachten gentechnisch veränderten Pflanzen etwa 1,4 Milliarden US\$. 1999 wurden weltweit auf 40 Mill. Hektar gentechnisch veränderte Pflanzen angebaut; in den USA sind 50\% der Gesamtanbaufläche für Sojabohnen, mehr als $30 \%$ der für Baumwolle und $20 \%$ der für Mais in diesem Jahr mit den entsprechend gentechnisch veränderten Pflanzen bestellt worden. In Deutschland zeigen sich indessen nur verhaltene Auswirkungen dieser Entwicklung $[1,2]$. Als ein Maß für den Entwicklungsstand der „Grünen Gentechnik” in der Pflanzenzüchtung [3] und damit u.a. auch in der Produktion von Futterund Lebensmitteln kann die Anzahl von Freisetzungsvorhaben mit gentechnisch veränderten Pflanzen gelten. Von den weltweit mehr als 5000 derartigen Freisetzungsvorhaben entfallen auf die EUMitgliedstaaten nahezu 1500 (Zeitraum von 1991 bis 1999). Im Vergleich mit den anderen EU-Mitgliedstaaten liegt Deutschland mit etwa 100 genehmigten Freisetzungsvorhaben auf dem 7. Platz und damit zwar auf einer mittleren Position; jedoch sollte nicht unbeachtet bleiben, daß 84\% der Freisetzungsvorhaben mit gentechnisch veränderten Pflanzen in nur sechs EU-Mitgliedstaaten (Frankreich, Italien, England, Spanien, den Niederlanden und Belgien) stattgefunden haben (Abb.1).

\section{Gentechnische Veränderungen aus fünf Anwendungsbereichen}

In den ersten Jahren nach 1991 fanden hauptsächlich Freisetzungsvorhaben mit gentechnisch veränderten Pflanzen statt, deren gentechnische Modifikation darauf abzielte, diese für agronomische Belange zu verbessern. $\mathrm{Zu}$ derartigen gentechnischen Veränderungen zählen z.B. die Etablierung von Toleranzen gegen bestimmte Herbizide, von Resistenzen gegen phytopathogene Organismen und die Einführung der männlichen Sterilität zur Erzeugung von Hybridsaatgut (Heterosiseffekt). Die Freisetzungsaktivitäten mit gentechnisch veränderten Pflanzen dieser Art haben auch in den

Prof.Dr. Dr. Peter Brandt

Robert Koch-Institut, Zentrum Gentechnologie, Postfach 6502 80, D-13302 Berlin,

E-Mail:pbrandt@rki.de. 
Bundesgesundheitsbl -

Gesundheitsforsch - Gesundheitsschutz

$2000 \cdot 43: 87-93$ @ Springer-Verlag 2000

\section{P. Brandt}

Transgenic plants of the second and third generation: What can we expect?

\section{Summary}

Genetically modified plants are classified referring to their genetical modifications and attached to five different ranges of applications. Whereas genetically modified plants of the range of application "Agricultural Requirements" (for example herbicide tolerance and insect resistance) already were tested in numerous experimental field releases within the area of the EU-memberstates and some of these genetically modified plants (as well as the processed products of them) have been already approved for placing on the market in the EU, genetically modified plants of the "Second and Third Generation" follow with a delay of some years. These genetically modified plants are belonging to the four ranges of applications "Endogenous Plant Stuff",'Novel Ingredients for Food Production,"' Pharmaceutical/Medical Applications" and "Applications for Chemical Industry".

But non only the palette of genetical modifications will be enlarged for genetically modified plants in the future and will be more concentrated to the four ranges of applications mentioned above, but also the genetic design of the transgenic plants will be further developed by using transformation of the chloroplasts, chimeraplasty and "Genetic Use Restriction Technology" (GURT).

\section{Key words}

Endogenous Plant Stuff · Novel Ingredients · Pharmaceutical/Medical Applications . Chimeraplasty · GURT · Genetic Use Restriction Technology

\section{Leitthema: Perspektiven zur Entwicklung der „Grünen Gentechnik"}

letzten Jahren nicht nachgelassen (Abb. 2)

Weitere Aspekte könnten in $\mathrm{Zu}$ kunft im Rahmen von Freisetzungsvorhaben mit gentechnisch veränderten Pflanzen erprobt werden, die bei den bisherigen Freisetzungsexperimenten des o.g. Anwendungsbereichs noch nicht vertreten waren. Dazu könnten zum Beispiel gentechnisch veränderte Pflanzen gehören, die aufgrund ihrer gentechnischen Modifikation auf Schwermetallbelasteten Böden wachsen können [4] bzw. tolerant sind gegenüber Kälte, Trockenheit oder Ozonbelastung. Das derartige Prognosen nicht nur hypothetischen Charakter haben, zeigen die Versuche von Rugh et al. [5], die Liriodendron tulipifera (Tulpenbaum) mit dem bakteriellen Gen merA transformierten. merA codiert für eine Quecksilber-Reduktase, die es den gentechnisch veränderten Tulpenbäumen ermöglicht, toxisches $\mathrm{Hg}^{2+}$ in das weniger toxische, relativ inerte metallische $\mathrm{Hg}^{\circ}$ umzuwandeln; dieses wird zum Teil von den gentechnisch veränderten Pflanzen in die Luft abgegeben. Derartige gentechnisch veränderte Pflanzen werden bereits kommerziell für die Sanierung von Quecksilber-verseuchten Böden in den USA angeboten.

\section{„Die Einsatzmöglichkeiten gentechnisch veränderter Pflanzen sind im Laufe der vergangenen Jahre sehr viel umfangreicher geworden."}

Um etwa drei Jahre versetzt gegenüber dieser ersten Gruppe von Freisetzungsvorhaben finden seit etwa 1995 verstärkt Freisetzungen mit gentechnisch veränderten Pflanzen statt, deren gentechnische Modifikationen darauf abzielen, ihre endogenen Inhaltsstoffe qualitativ oder quantitativ zu verändern. $\mathrm{Zu}$ derartigen gentechnischen Veränderungen zählen z.B. die Modifizierung des Fettsäuremusters oder der Kohlenhydratzusammensetzung (Abb. 2).

Aufgrund bereits publizierter Ergebnisse ist für die Zukunft zu erwarten, daß Freisetzungsvorhaben mit gentechnisch veränderten Pflanzen bevorstehen können, deren gentechnische Veränderungen noch ganz anderen Zwecken als den zuvor genannten dienen sollen. Es ist derzeit absehbar, daß in Zukunft gentechnisch veränderte Pflanzen aufgrund ihrer gentechnischen Veränderung in den folgenden fünf Bereichen Anwendung finden könnten (rev. 3):

1. Agronomischer Bedarf: z.B. HerbizidToleranz, Virus-Resistenz; Pilz-Resistenz; Bakterien-Resistenz; InsektenResistenz; Frosttoleranz; männliche Sterilität; Schwermetall-Toleranz; Ozon-Toleranz.

2. Endogene Inhaltstoffe: z.B. Veränderung der Blütenfarbe, des Fettsäuremusters, der Kohlenhydratzusammensetzung oder des Nitratstoffwechsels.

3. Neuartige Substanzen für die Lebensmittelproduktion: z.B. die Synthese von Fructanen [6] oder die Anreicherung ungesättigter Fettsäuren [7].

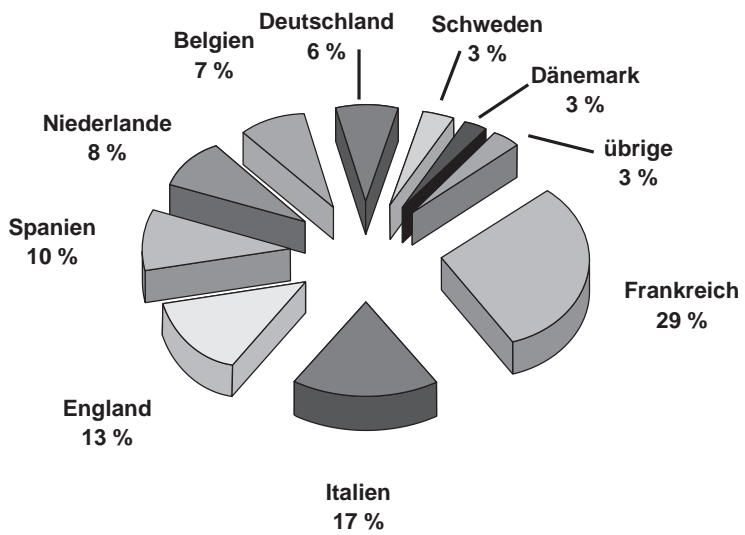

Abb. $1 \Delta$ Prozentuale Verteilung der Anmeldungen von Freisetzungsvorhaben mit gentechnisch veränderten Pflanzen im Europäischen Beteiligungsverfahren auf die EU-Mitgliedstaaten (übrige=Finnland, Griechenland, Portugal, Irland, Österreich) (Stand: November 1999; Quelle: http://www.rki.de) 


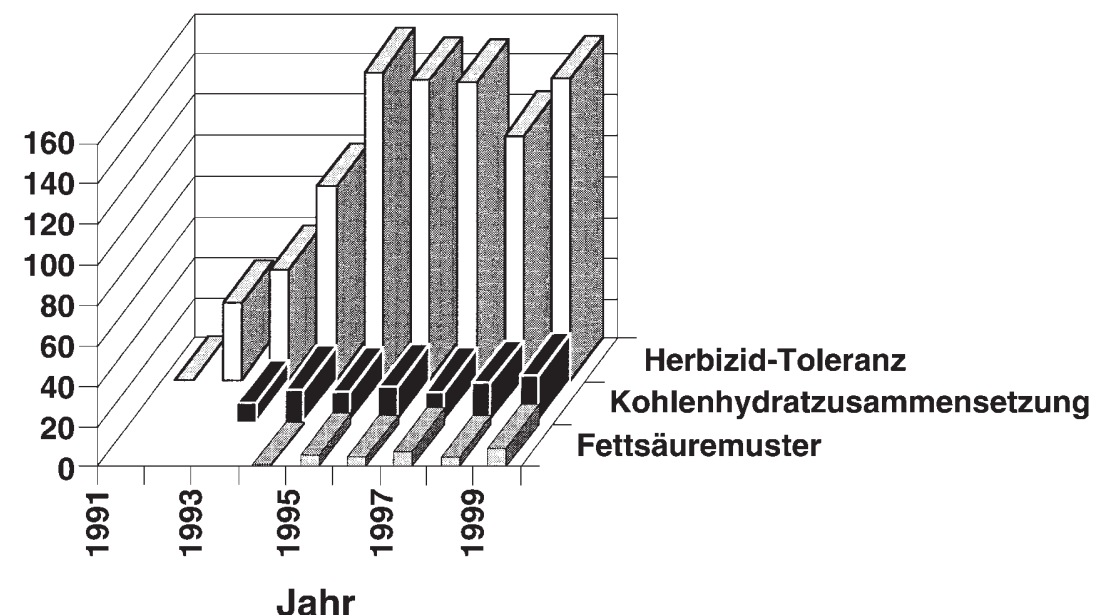

Abb. $2 \Delta$ Anzahl der Anmeldungen von Freisetzungsvorhaben mit gentechnisch veränderten Pflanzen (gentechnische Modifikation ist eine spezifische Herbizid-Toleranz oder betrifft die Kohlenhydratzusammensetzung bzw. das Fettsäuremuster) im Zeitraum von 1991 bis 1999 für den Bereich der EU-Mitgliedstaaten. Weitere Erläuterungen im Text (Stand: November 1999; Quelle: http://www.rki.de)
4. Pharmazeutische und/oder medizinische Anwendungen: z.B. die Synthese spezieller Proteine $[8,9,10]$ oder von „essbaren Impfstoffen” [11, 12] oder die Anreicherung von Metaboliten des pflanzlichen Stoffwechsels $[13,14]$.

5. Anwendungen für die chemische Industrie: z.B. die Synthese von Polyhydroxybuttersäure [15] oder die Anreicherung von Metaboliten des pflanzlichen Stoffwechsels [16].

Es kann auch für die Freisetzungsvorhaben mit gentechnisch veränderten Pflanzen der Anwendungsbereiche drei bis fünf erwartet werden, daß sie zuwendungsbereiche eins und zwei - jeweils zeitlich um einige Jahre gegeneinander versetzt - „wellenartig” einsetzen werden, wobei derzeit weder über den Zeitpunkt, wann derartige FreisetzunRealität werden sollten, noch über die Intensität und die Dauer der einzelnen „Wellen” etwas ausgesagt werden kann.

Liegt genügend Erfahrung aus etlichen Freisetzungsexperimenten mit gentechnisch veränderten Pflanzen in mehreren Jahren und an verschiedenen Standorten vor und haben sich die Erwartungen an die jeweilige gentechnisch veränderte Pflanze bestätigt [17], so wird in der Regel ein Antrag auf Genehmigung des „Inverkehrbringen” (d.h. kommerzieller Einsatz der jeweiligen genkünftig genauso wie schon die der Angen im Gebiet der EU-Mitgliedstaaten dungsbereich eins und drei dem Anwendungsbereich zwei zuzuordnen sind. die gentechnisch veränderten Pflanzen, die den Anwendungsbereichen drei bis fünf zuzurechnen sind, sich erst im Stadium der Laboruntersuchungen befinden und daß die Palette der Möglichkeiten für die Anwendungsbereiche eins und zwei keine weiteren Möglichkeiten mehr aufweist, seien exemplarisch einige zukunftsweisende Beispiele aufgeführt:

Die Etablierung der Resistenz gegen bestimmte phytopathogene Insekten in gentechnisch veränderten Pflanzen erfolgt derzeit fast ausschließlich mit Hilfe der Übertragung eines der $\delta$-Endotoxin-Gene aus Bacillus thuringiensis. Die Untersuchungen, mit Hilfe von verschiedener Amylase- oder Protease-Inhibitoren ebenfalls eine derartige InsektenResistenz in gentechnisch veränderten Pflanzen zu erzeugen [18], haben erst zum Teil das Stadium erreicht, Freisetzungsexperimente mit solchen gentechnisch veränderten Pflanzen vorzunehmen. Bowen et al. [19] haben kürzlich darauf hingewiesen, daß es möglich sein könnte, eine Insekten-Resistenz in Kulturpflanzen $\mathrm{zu}$ etablieren durch die
Um dem Eindruck vorzubeugen, daß

Tabelle 1

Produkte aus dem Bereich der ",Grünen Gentechnik", für die ein Inverkehrbringen in der Europäischen Union genehmigt wurde. GVO=gentechnisch veränderter Organismus; HAT= Herbizid-Toleranz; IR = Insekten-Resistenz; MS = Männliche Sterilität; VB = Veränderung der Blütenfarbe; VL = Verlängerung der Haltbarkeit als Schnittblume. (Quelle: http://www.rki.de)

\begin{tabular}{llll} 
Antragsteller & GVO & Gentechnische Veränderung & Genehmigt \\
\hline Seita & Tabak & HAT & 1994 \\
Plant Genetic Systems & Raps* $^{*}$ & MS, HAT & 1996 \\
Novartis & Mais & IR, HAT & 1997 \\
Bejo Zaden BV & Radicchio & MS & 1996 \\
Monsanto & Soja & HAT & 1996 \\
Plant Genetic & a) Raps* & MS, HAT & 1997 \\
Systems & b) Raps* & MS, HAT & 1997 \\
AgrEvo & Raps & HAT & 1998 \\
AgrEvo & Mais & HAT & 1998 \\
Monsanto & Mais & IR & 1998 \\
Northrup & Mais & IR & 1998 \\
Florigene & Nelke** & VB & 1998 \\
Florigene & Nelke & VL & 1998 \\
Florigene & Nelke** & VB & 1998 \\
\hline
\end{tabular}

${ }^{*} b z w .{ }^{* *}=$ gentechnisch veränderte Pflanzen, die aus verschiedenen Transformationsexperimenten hervorgegangen sind und deren Inverkehrbringen daher auch separat beantragt und genehmigt wurde 
Transformation mit den Genen $t c a, t c b$, tcc und/oder tcd aus Photorhabdus luminescens, die für die Untereinheiten eines auf Insekten wirkenden Toxins codieren.

Mittels gentechnischer Methoden können Pflanzen dergestalt transformiert werden, daß sie - unter der Voraussetzung, daß ein geeignetes Genkonstrukt verwandt worden ist - grundsätzlich jedes „Fremd”-Protein synthetisieren und anreichern können (Tabelle 2). Diese Anwendung, die dem Bereich vier zugerechnet werden kann, hat in einzelnen Fällen schon den Grad der Kommerzialisierung erreicht. So haben Hood et al. [9] Mais mit dem Gen für das Avidin (einem Glycoprotein, das in den Eiern von Vögeln, Amphibien und Reptilien vorkommt) transformiert. Dieses Glycoprotein ist in den Maiskörnern nachweisbar mit einer Menge von $230 \mathrm{mg} / \mathrm{kg}$ Saatgut. Die Extraktion des Avidin aus gentechnisch veränderten Maiskörnern hat sich in technischer Hinsicht als leichter erwiesen als aus Hühnereiern; sowohl quantitativ (Ausbeute von fast $100 \%$ ) als auch qualitativ ist das gentechnisch veränderte Maissaatgut besser zur Gewinnung von Avidin geeignet. Hood et al. [9] verweisen darauf, daß zur Gewinnung von $20 \mathrm{~g}$ Avidin nur $100 \mathrm{~kg}$ gentechnisch veränderte Maiskörner, aber 900 kg Hühnereier benötigt werden. Das aus gentechnisch veränderten Maiskörnern gewonnene Avidin wird schon kommerziell als Substanz für diagnostische Zwecke angeboten.

Unter dem Anwendungsbereich vier sind auch die sogenannten ,essbaren Impfstoffe" aufgeführt. Die Bezeichnung „essbare Impfstoffe” mag manchem noch unwahrscheinlich vorkommen; die experimentellen Erfolge auf Laborniveau sprechen allerdings dafür, dass diese in situ - Anwendung von Antigen- bzw. "Plantibody"-produzierenden Kulturpflanzen in naher Zukunft Realität werden könnte (Tabelle 3; rev. 11). In den Medien wird bisweilen schon jetzt über die Etablierung entsprechend gentechnisch veränderter Pflanzen berichtet, die herkömmlich roh als Obst oder Salat gegessen werden (z.B. Tomaten, Avocado oder Bananen), und durch deren Verzehr Immunität gegen bestimmte Krankheitserreger erreicht werden soll.

\section{Möglichkeiten des genetischen Design zukünftiger gentech- nisch veränderter Pflanzen}

Bei dieser reichhaltigen Palette möglicher zukünftiger gentechnisch veränderter Pflanzen (für die in den folgenden Beiträgen dieses Heftes einige Beispiele vorgestellt werden $[4,6,8,12,13,14$, 16] sollte nicht der Blick für Neuerungen in Bezug auf das veränderte genetische Design dieser Pflanzen verloren gehen, das sich in wesentlichen Aspekten von dem der heutigen gentechnisch veränderten Pflanzen unterscheiden könnte.

In dieser Hinsicht sind zum Beispiel die Untersuchungen von Daniell et al. [33] zur Transformation des Plastoms von Tabakpflanzen von besonderem Interesse. Sie verwenden dazu ein chimäres Genkonstrukt aus dem Gen für die 5Enol-Pyruvyl-Shikimat-3-PhosphatSynthase (EPSPS) aus Petunia hybrida, das flankiert wird von dem plastidären Gen für die Große Untereinheit der Carboxydismutase und dem plastidären ORF12. Diese gentechnisch veränderten Tabakpflanzen besitzen durch die Transformation des Plastoms pro Zelle 5000 bis 10 ooo Kopien des integrierten Genkonstrukts. Dies bewirkt eine erhöhte Expressionsrate der EPSPS und damit eine effektivere Resistenz gegenüber der Behandlung mit dem Herbizid Roundup. Durch die Verwendung der beiden plastidären Gene als Border ist bei dieser Transformationsweise vorherbestimmbar, an welchem Ort auf dem Plastom das Genkonstrukt integriert wird. Ferner wird durch die Beschränkung der gentechnischen Veränderung auf das Plastom eine Art biologisches Con- tainment erreicht, da beim Tabak wie auch bei anderen Kulturpflanzen die Pollen keine Plastiden enthalten und somit auch die Übertragung der gentechnischen Veränderung auf nicht-gentechnisch veränderte verwandte Pflanzen beim Auskreuzen über die Pollen nicht erfolgen kann.

Einen entscheidenden Schritt weiter geht die „Chimeraplastie” genannte Methode, mit deren Hilfe kürzlich in $\mathrm{Ni}$ cotiana tabacum [34] bzw. in Zea mays [35] jeweils eine Herbizid-Toleranz gegen Imidazolin bzw. Sulfonyl-HarnstoffHerbizide etabliert werden konnte. Anstelle der Insertion eines vollständigen Gen(konstrukt)s in das pflanzliche Genom - wie es bei den herkömmlichen Transformationsmethoden üblich ist werden bei der Chimeraplastie chimäre Oligonucleotide (COs) eingesetzt, die (im vorliegenden Fall) aus einer fünf Nucleotide umfassenden DNA-Sequenz zwischen zwei jeweils zehn Nucleotide umfassende RNA-Sequenzen bestehen. Innerhalb der DNA-Sequenz unterscheidet sich ein Nucleotid von dem in der entsprechenden DNA-Sequenz des Zielgens der $\mathrm{zu}$ transformierenden Pflanze. Nach Einbringen der COs in die pflanzlichen Zellen mittels „Partikel-Beschuß" kommt es zur homologen Rekombination zwischen CO und Zielgen und - vermittelt durch zelleigene Reparaturmechanismen - zur Übernahme der Nucleotidveränderung der DNA-Sequenz des CO in die Sequenz des Zielgens. Noch ist derzeit die Effektivität der Chimeraplastie gering; ihre Präzision bei der punktuellen Veränderung des pflanzlichen Genoms läßt jedoch jetzt schon erwarten, daß diese

\begin{tabular}{|c|c|c|}
\hline Protein & $\begin{array}{l}\text { Herkunft der entsprechenden } \\
\text { DNA-Sequenz }\end{array}$ & $\begin{array}{l}\text { Gentechnisch veränderte } \\
\text { Pflanze }\end{array}$ \\
\hline Avidin [9] & Huhn & Zea mays \\
\hline$\alpha$-Amylase [20] & Bacillus licheniformis & Vicia narbonensis \\
\hline Phytase [21] & Aspergillus niger & Nicotiana tabacum \\
\hline$(1,3-1,4)-\beta$-Glucanase [22] & Bacillus amyloliquefaciens & Hordeum vulgare \\
\hline$(1,4)-\beta$-Xylanase [23] & Ruminococcus flavefaciens & Nicotiana tabacum \\
\hline Lactoferrin [8] & Homo sapiens & Nictotiana tabacum \\
\hline
\end{tabular}


Tabelle 3

Auswahl bislang publizierter Arbeiten zur Erzeugung von Antikörpern gegen Krankheitserreger oder von deren Antigenen mit Hilfe von entsprechend transformierten Pflanzen ( $t$ ) bzw. von Impfstoffen mittels chimärer Viren nach deren Vervielfältigung in infizierten Pflanze (i) [verändert nach 11]

\begin{tabular}{|c|c|c|c|}
\hline Jahr & Krankheitserreger & $\begin{array}{l}\text { Zur Transformation } \\
\text { verwendete Nukleinsäure } \\
\text { kodiert für }\end{array}$ & $\begin{array}{l}\text { Transformierte (t) } \\
\text { bzw. infizierte } \\
\text { (i) Pflanze }\end{array}$ \\
\hline 1992 & $\begin{array}{l}\text { Hepatitis B Virus } \\
\text { [24] }\end{array}$ & $\begin{array}{l}\text { Hüllprotein } \\
\text { des Hepatitis B Virus }\end{array}$ & Nicotiana tabacum $(\mathrm{t})$ \\
\hline 1993 & $\begin{array}{l}\text { Foot-and-mouth- } \\
\text { disease Virus } \\
{[25]}\end{array}$ & $\begin{array}{l}\text { chimäres Hüllprotein des Cowpea } \\
\text { mosaic virus mit Epitopbereich des } \\
\text { Foot-and-mouth-disease virus }\end{array}$ & Vigna ungiuculata (i) \\
\hline 1994 & $\begin{array}{l}\text { HIV,Typ } 1 \\
{[26]}\end{array}$ & $\begin{array}{l}\text { chimäres Hüllprotein } \\
\text { des Cowpea mosaic virus } \\
\text { mit Epitopbereich des HIV-1 }\end{array}$ & Vigna unguiculata (i) \\
\hline 1994 & $\begin{array}{l}\text { humanes Rhinovirus } \\
\text { [26] }\end{array}$ & $\begin{array}{l}\text { chimäres Hüllprotein } \\
\text { des Cowpea mosaic virus } \\
\text { mit Epitopbereich des HTV-14 }\end{array}$ & Vigna unguiculata (i) \\
\hline 1994 & $\begin{array}{l}\text { Streptococcus mutans } \\
\text { [27] }\end{array}$ & $\begin{array}{l}\text { Antikörper gegen Hüllprotein } \\
(185 \mathrm{kDa}) \text { von S. mutans }\end{array}$ & Nicotiana tabacum $(\mathrm{t})$ \\
\hline 1995 & $\begin{array}{l}\text { Plasmodium malariae } \\
\text { [28] }\end{array}$ & $\begin{array}{l}\text { chimäres Hüllprotein } \\
\text { des Tobacco mosaic virus mit } \\
\text { Epitopbereich von P. malariae }\end{array}$ & Nicotiana tabacum (i) \\
\hline 1995 & $\begin{array}{l}\text { E. coli } \\
{[29]}\end{array}$ & $\begin{array}{l}\text { Untereinheit }(11,6 \mathrm{kDa}) \\
\text { des Hitze-instabilen } \\
\text { Enterotoxins von E. coli }\end{array}$ & $\begin{array}{l}\text { Nicotiana tabacum }(\mathrm{t}) \\
\text { Solanum tuberosum }(\mathrm{t})\end{array}$ \\
\hline 1996 & $\begin{array}{l}\text { Norwalk Virus } \\
{[30]}\end{array}$ & $\begin{array}{l}\text { virales Kapsid-Protein ( } 58 \mathrm{kDa} \text { ) } \\
\text { des Norwalk Virus }\end{array}$ & $\begin{array}{l}\text { Nicotiana tabacum }(\mathrm{t}) \\
\text { Solanum tuberosum }(\mathrm{t})\end{array}$ \\
\hline 1997 & $\begin{array}{l}\text { Mink enteritis Virus } \\
\text { [31] }\end{array}$ & $\begin{array}{l}\text { chimäres Hüllprotein des Cowpea } \\
\text { mosaic virus mit Epitopbereich des } \\
\text { VP2 Kapsid-Proteins des MEV }\end{array}$ & Vigna unguiculata (i) \\
\hline 1999 & $\begin{array}{l}\text { Cytomegalovirus } \\
\text { [32] }\end{array}$ & $\begin{array}{l}\text { Glycoprotein B (UL55) des } \\
\text { Cytomegalovirus }\end{array}$ & Nicotiana tabacum $(\mathrm{t})$ \\
\hline
\end{tabular}

Methode zukünftig an Bedutung gewinnen könnte. Es bleibt allerdings auch zu klären, ob es sich bei dieser Methode überhaupt um ein gentechnisches Verfahren im Sinne des GenTG handelt?

\section{„Zukünftige gentechnisch verän- derte Pflanzen werden sich in wesentlichen Aspekten durch das veränderte genetische Design von den heutigen unterscheiden."}

Viel Anlaß zu gesellschaftlichem Disput hat vor einiger Zeit die Patentierung der in den Medien als „Terminator”-Technik bezeichneten gentechnischen Veränderung gegeben [36]; es handelt sich dabei um eine Variante der „Genetic Use
Restriction Technology” (GURT). Prinzipiell besteht das patentierte „System zur Kontrolle der pflanzlichen Genexpression" - wie es in der Patentschrift heißt - aus drei verschiedenen Genkonstrukten (Abb.3), deren Zusammenwirken dazu führen soll, daß es mit dem vom Saatguthersteller ausgeliefertem Saatgut zwar möglich ist, am Ende der ersten Vegetationsperiode in herkömmlicher Weise eine Ernte einzubringen, $\mathrm{da}$ aber das Erntegut für eine weitere Aussaat in der nächsten Vegetationsperiode nicht mehr verwendet werden kann, da - aufgrund der gentechnischen Veränderung - die Keimfähigkeit des geernteten Saatgutes vernichtet worden ist. Es wird davon ausgegangen, daß die praktische Umsetzung dieses Konzeptes mindestens noch vier bis fünf Jahre in Anspruch nehmen würde, wobei heute schon gemutmaßt werden kann, ob es jemals gelingen sollte, daß das Zusammenspiel der drei eingebrachten Fremdgene auf die o.g. Weise in allen geernteten Samen der gentechnisch veränderten Pflanzen (d.h. zu 100\%) erfolgt. So nimmt es nicht Wunder, daß kürzlich von der maßgeblichen Firma verkündet wurde, daß die Umsetzung des Patents in die Anwendung nicht weiter verfolgt werden soll.

Durch die Mediendiskussion über die umstrittene „Terminator”-Technik ist eine Entwicklungsmöglichkeit der GURT für die „Grüne Gentechnik” bislang in der Öffentlichkeit wenig beachtet worden. Wenn es im Verlauf der zahlreichen Genom-Projekte gelingen sollte, sehr spezifische Promotoren zu identifizieren, die (im Idealfall ausschließlich) durch Einwirken pflanzenexterner, sehr spezieller Inducer ,zum Anschalten” ihrer nachgeordneten, codierenden DNASequenz veranlaßt werden können, so wäre die Etablierung gentechnisch veränderter Pflanzen möglich, deren gentechnische Veränderung auf diese Weise z.B. aufgrund der aktuellen Witterungsbedingungen ,,an- bzw. abgeschaltet” werden könnten.

Ebenso aus öffentlichen Diskussionen und aus den Berichten in den Medien ist hinlänglich bekannt, daß in vielen Fällen Antibiotika-Resistenzgene als Marker-Gene bei der Transformation von Pflanzen verwendet werden [37]. In den letzten Jahren wurden jedoch auch Verfahren entwickelt, welche die Etablierung gentechnisch veränderter Pflanzen ohne Marker-Gene grundsätzlich möglich machen [z.B. 38, 39, 40]; es ist aber durchaus möglich, daß im konkreten Einzelfall aufgrund der Eigenschaften der Kulturpflanze, die transformiert werden soll, diese Methoden nicht einsetzbar sind. Diese Transformationsverfahren, die im Ergebnis zu Markergenfreien gentechnisch veränderten Pflanzen führen, sind u.a. auch deswegen von Interesse, weil es experimentell nur so möglich ist, in aufeinanderfolgenden, unabhängigen Transformationsschritten mehrere verschiedene Genkonstrukte in die Primärtransformante zu integrieren. In den nächsten Jahren wird sich zeigen, ob sich diese Tranformationsverfahren ohne den Einsatz von Antibiotika-Resistenzgenen als Marker bewähren werden. 


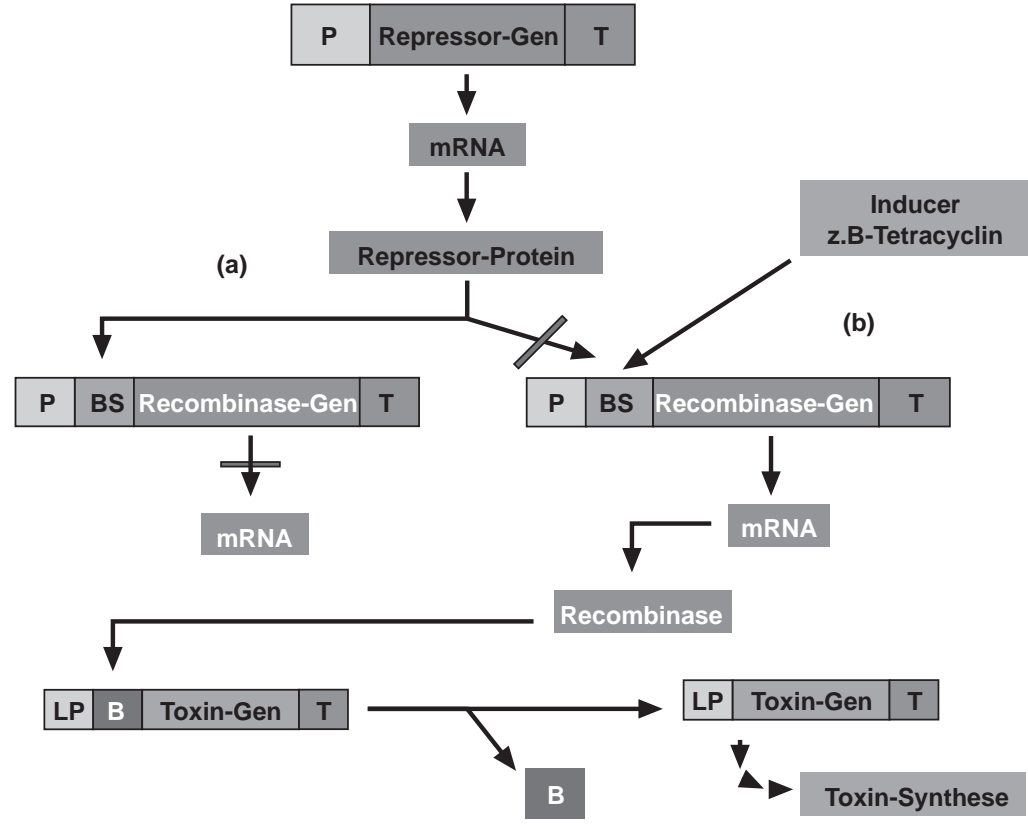

Abb. $3 \Delta$ Schematische Darstellung des Zusammenwirkens von drei in das Pflanzengenom integrierten Genkonstrukten zur Verhinderung der erneuten Verwendung geernteter Samen als Saatgut (,Teminator"-Technik). Während der Saatgutgewinnung (a) bindet das exprimierte Repressor-Protein am Recombinase-Gen und verhindert auf diese Weise dessen Expression. Soll das Saatgut in den Handel gebracht werden, wird es mit einem Inducer behandelt (b), der die Bindung des Repressor-Proteins am Recombinase-Gen verhindert. Dadurch kommt es zur Expression des Recombinase-Gens und die Recombinase kann einen Blocker aus dem Toxin-Gen entfernen. Dessen für die späte Entwicklungsphase des pflanzlichen Embryos spezifischer Promoter läßt die Expression des Toxin-Gens erst in diesem Entwicklungsstadium des Samens zu; das synthetisierte Toxin unterbindet die Embryonalentwicklung und damit die Keimfähigkeit des Samens

\section{Monitoring und Information}

Es ist sicher unangemessen, in Euphorie über die zukünftigen Möglichkeiten, gentechnisch veränderte Pflanzen für ganz spezielle Bedürfnisse kreieren und etablieren zu können, die notwendigen Sicherheitsbelange außer Acht zu lassen. Die zukünftige Weiterentwicklung gentechnischer Modifikationen für die o.g. Anwendungsbereiche bringt es zum Beispiel sicher mit sich, dass - im Gegensatz zu heutigen gentechnischen Veränderungen - oft mehr als nur ein „Fremdgen” in das pflanzliche Genom integriert werden wird und dass dadurch komplexere Auswirkungen auf die pflanzlichen Stoffwechselwege zu erwarten sein können. Es steht außer Frage, daß die zuständigen Behörden schon jetzt auf diese mögliche Weiterentwicklung der „Grünen Gentechnik” vorbereitet sein müssen, um bei Sicherheitsbewertungen der zukünftigen gentech- nisch veränderten Pflanzen den gesetzlichen Auftrag erfüllen zu können.

In diesem Sinne sind auch die derzeitigen Bestrebungen zu sehen, ein anbaubegleitendes Monitoring für gentechnisch veränderte Pflanzen einzuführen, deren Inverkehrbringen für den $\mathrm{Be}$ reich der EU-Mitgliedstaaten entsprechend genehmigt worden ist (Tabelle 1). Bei dem anbaugegleitendem Monitoring sieht das Robert Koch-Institut als Genehmigungsbehörde seine Aufgabe u.a. darin, genehmigungsrelevante Informationen zu sammeln, zu bewerten und für Interessierte zur Verfügung zu stellen. Im Rahmen des Gesamtkonzepts (über das zu einem späteren Zeitpunkt berichtet werden wird) ergibt sich für das Robert Koch-Institut, aufgrund der jeweiligen Datenlage laufende Monitoringvorhaben in geeigneter Weise zu modifizieren bzw. neue Monitoringvorhaben zu initiieren. Es ist vorstellbar, daß zukünftige Monitoringvorhaben entweder grundsätzlichen Fragestellungen nachgehen oder aber von der Sache her eng verknüpft mit dem kommerziellen Anbau einer bestimmten gentechnisch veränderten Pflanze sind. Zu diesem Zweck beabsichtigt das Robert Koch-Institut eine „Zentrale Koordinationsstelle für das anbaubegleitende Monitoring von gentechnisch veränderten Pflanzen" einzurichten, dem ein „Genregister” für Nachweisfahren im Rahmen des anbaubegleitenden Monitoring beigeordnet sein soll.

\section{Literatur}

1. Brandt $P$ (1997) Zukunft der Gentechnik. Birkhäuser-Verlag, Basel, S 290

2. Brandt $P$ (1999) Was wird aus der Gentechnik? Forschung \& Lehre, Heft 11, S 570-572

3. Brandt $P$ (1995) Gentechnisch veränderte Pflanzen. Herstellung, Anwendung, Risken und Richtlinien. Birkhäuser-Verlag, Basel, S 306

4. Liu JR, Suh MC, Choi D (2000) Phytoremediation of cadmium contamination: Overexpression of metallothionein in transgenic tobacco plants. Bundesgesundheitsbl 43: 126-130

5. Rugh CL, Senecoff JF, Meagher RB, Merkle SA (1998) Development of transgenic yellow poplar for mercury phytoremediation. Nature Biotechnology 16:925-928

6. Heyer A (2000) Production of modified carbohydrates in transgenic plants. Bundesgesundheitsbl 43:94-98

7. Murphy DJ (1999) Production of novel oils in plants. Curr Opinion Biotechnol 10: 175-180

8. Spik G, Theisen M (2000) Alteration of glycosylation of human lactoferrin expressed in transgenic tobacco. Bundesgesundheitsbl 43:104-109

9. Hood EE, Witcher DR, Maddock S, Meyer T, Baszczynski C, Bailey M, Flynn P, Register J, Marshall L, Bond D, Kulisek E, Kusnadi A, Evangelista R, Nikolov Z, Wooge C, Mehigh RJ, Hernan R, Kappel WK, Titland D, Li CP, Howard JA (1997) Commercial production of avidiv from transgenic maize: characterization of transformant, production, processing, extraction and purification. Molecular Breeding 3:291-306

10. Herbers K, Sonnewald U (1999) Production of new/modified proteins in transgenic plants. Curr Opinion Biotechnol 10:163-168

11. Brandt $P$ (1998) Impfschutz durch den Verzehr von gentechnisch verändertem 0 bst oder Gemüse? Bundesgesundheitsbl 41: 289-293 
12. Befus N, Langridge HR (2000) Plant-based cholera toxin B subunit-insulin/GAD fusion proteins suppress the development of autoimmune diabetes. Bundesgesundheitsbl 43:\&ding7A;

13. Kumar A (1998) Transgenic manipulation of polyamine metabolism. In: Lindsey K (ed) Transgenic plant research. Harwood Academic Publishers, pp 187-199

14. Napier JA (2000) Transgenic plants for the production of pharmaceutical fatty acids. Bundesgesundheitsbl 43:99-103

15. Poirier Y (1999) Production of new polymeric compounds in plants. Curr Opinion Biotechnol 10:181-185

16. Kopriva S, Rennenberg H (2000) Forest biotechnology and environment. Bundesgesundheitsbl 43:121-125

17. Brandt $P$ (1999) Prinzipien der Risikoerwägungen zur „Grünen Gentechnik". Bundesgesundheitsbl 42: (in press)

18. Jouanin L, Bonade-Bottino M, Girard C, Morrot G, Giband M (1998) Transgenic plants for insect resistance. Plant Science 131:1-11

19. Bowen D, Rochelaeu TA, Blackburn M, Andreev 0, Golubeva E, Bhartia R, French-Constant RH (1998) Insecticidial toxins from the bacterium Photorhabdus /uminescens. Sciene 280:2129

20. Pen J, Molendijk L, Quax WJ, Sijmons PC, van Ooyen AJJ, van den Elzen PJM, Rietveld K, Hoekema A (1992) Production of active Bacillus licheniformis alpha-amylase in tobacco and ist application in starch liquefaction. Bio/Technol 10:292-296

21. Verwoerd TC, van Paridon PA, van Ooyen AJJ, van Lent JWM, Hoekema A, Pen A (1995) Stable accumulation of Aspergillus niger phytase in transgenic tobacco leaves. Plant Physiol 109:1199-1205
22. Jensen LG, Olsen 0, Kops O, Wolf N, Thompsen KK, von Wettstein D (1996) Transgenic barley expressing a protein-engineered, thermostable $(1,3-1,4)-\beta$-glucanase during germination. Proc Natl Acad Sci USA 93: 3487-3491

23. Herbers K, Flint HJ, Sonnewald U (1996) Apoplastic expression of the xylanase and $\beta(1-3,1-4)$ glucanase domains of the xynD gene from Ruminococcus flavefaciens leads to funktional polypeptides in transgenic plants. Mol Breeding 2:81-87

24. Mason HS, Lam DM, Arntzen CJ (1992) Expression of hepatitis $B$ surface antigen in transgenic plants. Proc Natl Acad Sci USA 89: 11745-11749

25. Usha R, Rohll JB, Spall VE, Shanks M, Maule AJ, Johnson JE, Lomonosoff GP (1993) Expression of an animal virus antigenic site on the surface of a plant virus particle. Virol 197: 366-374

26. Porta C, Spall VE, Loveland J, Johnson JE, Barker PJ, Lomonosoff GP (1994) Development of cowpea mosaic virus as a high-yielding system for the presentation of foreign peptides. Virol 202: 949-955

27. Ma JK, Lehner T, Satbila P, Fux Cl, Hiatt A (1994) Assembly of monoclonal antibodies with IgGI and IgA heavy chain domains in transgenic tobacco plants. Eur J Immunol 24:131-138

28. Turpen TH, Reini SJ, Charoenvit Y, Hoffman SL, Fallarme V, Grill LK (1995) Malarial epitopes expressed on the surface of recombinant tobacco mosaic virus. Nature Biotechnol 13 53-57

29. Haq TA, Mason HS, Clements JD, Arntzen JT (1995) Oral immunization with a recombinant bacterial antigen produced in transgenic plants. Science $268: 714-716$

30. Mason HS, Ball JM, Shi JJ, Ji-ang X, Estes MK, Arntzen CJ(1996) Expression of Norwalk virus capsid protein in transgenic tobacco and potato and its oral immunogenicity in mice.Proc Natl Acad Sci USA 93:5335-5340

31. Dalsgaard K, Uttenthal A, Jones TD, Xu F, Merryweather A, Hamilton WDO, Langeveld, JPM, Boshuizen RS, Kamstrup S, Lomonosoff GP, Porta C, Vela C, Casal Jl, Meloen RH, Rodgers PB (1997) Plant-derived vaccine protects target animals against a viral disease. Nature Biotechnol 15:248-252
32. Tackaberry ES, Dudani AK, Prior F, Tocchi M, Sardana R, Altosaar I, Ganz PR (1999) Development of biopharmaceuticals in plant expression systems: cloning, expression and immunological reactivity of human cytomegalovirus glycoprotein B (UL55) in seeds of transgenic tobacco. Vaccine 17 : 3020-3029

33. Daniell $H$, Datta R, Varma S, Gray S, Lee SB (1998) Containment of herbicide resistance through genetic engineering of the chloroplast genome. Nature Biotechnol 16: 345-348

34. Beetham PR, Kipp PB, Sawyxky XL, Arntzen CJ, May GD (1999) A tool for functional genomics: Chimeric RNA/DNA oligonucleotides cause in vivo gene-specific mutations. Proc Natl Acad Sci USA 96:8774-8778

35. Zhu T, Peterson DJ, Tagliani L, St. Clair G, Baszczynski CL, Bowen B (1999) Targeted manipulation of maize genes in vivo using chimeric RNA/DNA oligonucleotides. Proc Natl Acad Sci USA 96:8768-8773

36. US-Patent Nr. 5723765 : Control of plant gene expression.

37. Brandt $P$ (1999) Antibiotika-Resistenzgene als Marker in gentechnisch veränderten Pflanzen. Bundesgesundheitsbl 42:51-57

38. Joersbo M, Okkels FT (1997) A novel principle for selection of transgenic plant cells: Positive selection. Plant Cell Reports 16 : 219-221

39. Yoder J,Gouldsbrough AP (1994) Transformation systems for generating markerfree transgenic plants. Nature Biotechnol 12:263-267

40. Ebinuma H, Sugita K, Matsunaga E, Yamakado M (1997) Selection of marker-free transgenic plants using the isopentenyl transferase gene. Proc Nat Acad Sci USA 94:2117-2121 